\title{
Control of $\mu$-Autonomous Surface Vehicle Using Sliding Mode Control
}

\author{
Yamato Kawamura, ${ }^{1}$ Junichiro Tahara, ${ }^{1 *}$ Tetsu Kato, ${ }^{1}$ \\ Shoichiro Baba, ${ }^{2}$ and Masakazu Koike ${ }^{1}$ \\ ${ }^{1}$ Tokyo University of Marine Science and Technology, \\ 2-1-6 Etchujima, Koto-ku, Tokyo 135-8533, Japan \\ ${ }^{2}$ Japan Agency for Marine-Earth Science and Technology, \\ 2-2-8 Natsushimacho,Yokosuka-shi, Kanagawa 237-0061, Japan
}

(Received September 30, 2019; accepted November 5, 2019)

Keywords: sliding mode control, autonomous surface vehicle, system identification, robot operating system

An autonomous surface vehicle (ASV) is a small autonomous boat that is expected to be used for automated investigations in radiation-contaminated areas or for unattended fishing, which will help the fishing industry, whose workforce is significantly decreasing. However, the discussion on the modeling and control of an ASV has mainly been based on those of a conventional ship. We have developed a micro-ASV ( $\mu-\mathrm{ASV})$ on the basis of the concept that an ASV is a mobile robot. The translational motion of the $\mu$-ASV is controlled by main thrusters, but its rotation is controlled by a bow thruster, not by a rudder as in an ordinary ship. Using the robot operating system (ROS), we demonstrated that the models of translational and rotational motions can be easily identified by adopting a distributed design using sliding mode control, in which the motion of the $\mu$-ASV is controlled by main and bow thrusters. We also demonstrated that the translational and angular motions can be controlled by a simple dynamic positioning system (DPS). These findings allowed us to make the equations of motion and the control design of the $\mu$-ASV simpler than those of an ordinary ship. A manual operation test using a joystick was conducted in the open sea. Promising results were achieved and are presented in this paper.

\section{Introduction}

In recent years, there has been an increase in the development of autonomous surface vehicles (ASVs) or unmanned surface vehicles (USVs). These vehicles are expected to be used by the military and in marine and environment investigations. The size and configuration of an ASV vary from those of a small motorboat to those of a radio-controlled boat depending on the environment of usage and the intended use. In this study, we created a micro-ASV ( $\mu$-ASV) $2 \mathrm{~m}$ in length and $45 \mathrm{~kg}$ in weight that is expected to be used for investigations in dams and harbors or for fishery survey (Fig. 1). The ultimate objective for the $\mu$-ASV is to conduct *Corresponding author: e-mail: jtahar0@kaiyodai.ac.jp https://doi.org/10.18494/SAM.2019.2644 


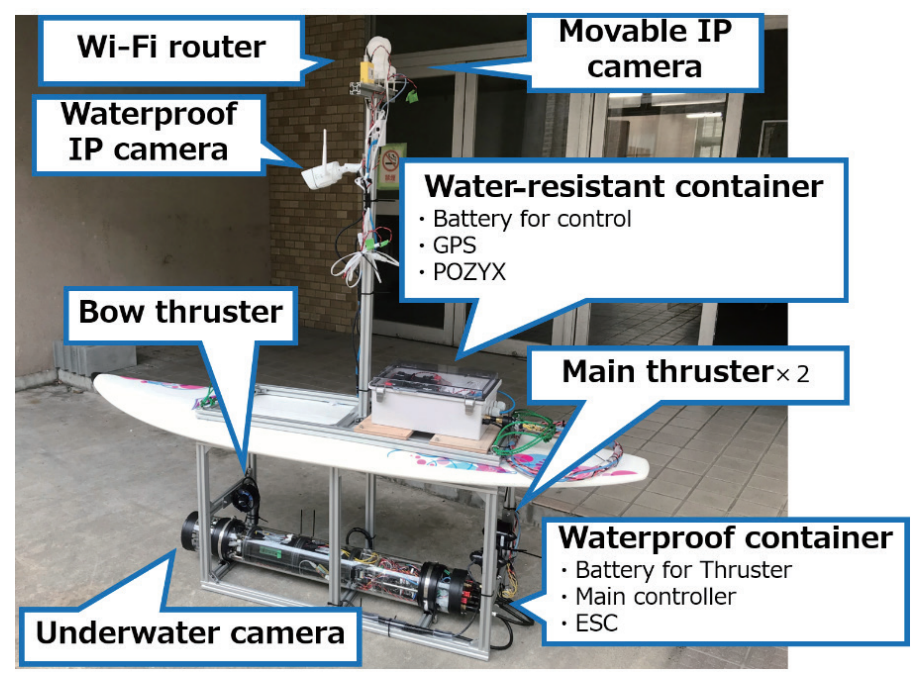

Fig. 1. (Color online) Basic $\mu$-ASV system.

automated investigations in radiation-contaminated areas of Fukushima Prefecture as well as investigations of barren areas caused by a huge outbreak of sea urchin in Miyagi Prefecture. Advanced control techniques, such as control with a dynamic positioning system (DPS) and heading control, are required to conduct these missions. Also, the accurate identification of the models of the $\mu$-ASV and the appropriate design of its control system are the basis for realizing these advanced control techniques. However, most ship model parameters are nonlinear and are strongly affected by water surface conditions. Although various modeling and control methods have been proposed, the models discussed in most studies are complicated and difficult to deal with. ${ }^{(1-3)}$ Also, an ASV must be tested in the sea. The modeling and construction of a control system are very difficult because the ASV is affected by sea conditions (waves and wind). ${ }^{(4)}$ In this study, these issues were resolved by employing the following three techniques:

1. To simplify the identification of control models, the motion models were simply calculated on the basis of the step responses from the forward propulsion thrusters (main thrusters) and those from the rotational thruster (bow thruster). ${ }^{(5-7)}$

2. Sliding mode control was used to make the control system less affected by model errors. The adoption of sliding mode control makes the control system simple and robust. ${ }^{(4,8)}$

3. To assess the validity of techniques (1) and (2), a simulation of the $\mu$-ASV was conducted on the robot operating system (ROS) with the thrusters replaced with omni wheels. ${ }^{(9,10)}$

The $\mu$-ASV was developed and tested using the techniques described above.

\section{2. $\mu$-ASV System}

The $\mu$-ASV is a surfboard equipped with two main thrusters for forward propulsion and a bow thruster. Unlike in an ordinary ship, the rotation angle of the $\mu$-ASV is not controlled by the difference between the output of the rudder and that of the main thrusters. ${ }^{(1)}$ Rather, the $\mu$-ASV resembles a mobile truck robot. The data from the CPU and sensors on the $\mu$-ASV are 
sent and received via Wi-Fi using a PC in a land station. At present, the sensor data from the $\mu$-ASV are analyzed at a land station to control the thrusters. Figure 1 shows a schematic of the $\mu$-ASV system and Table 1 shows the specifications of the $\mu$-ASV. Pixhawk, a simple inertial measurement unit (IMU) often used in a drone, is used as an accelerometer. As shown in the system configuration diagram (Fig. 2), the Li-ion battery can be charged wirelessly without opening the pressure-resistant container. In the design concept, the battery for the thrusters and the controller are placed in the pressure-resistant container underwater.

Table 1

$\mu$-ASV specifications.

\begin{tabular}{|c|c|c|c|}
\hline \multirow{2}{*}{$\begin{array}{l}\text { Metric } \\
\text { Body }\end{array}$} & & \multicolumn{2}{|r|}{ Specifications } \\
\hline & & Size & $1950 \times 530 \times 1650(\mathrm{~mm})$ \\
\hline & & Weight & about $45 \mathrm{~kg}$ \\
\hline \multirow[t]{2}{*}{ Thruster } & & Model & T200thruster \\
\hline & & Max Power & $350 \mathrm{~W}$ \\
\hline \multirow[t]{3}{*}{ Measuring instrument } & & IMU sensor & Pixhawk \\
\hline & & GPS & Ublox NEO M8N GPS For APM and Pixhawk \\
\hline & & Position & POZYX \\
\hline \multirow[t]{4}{*}{ Battery for thruster } & & Type & Lithium-ion battery \\
\hline & $\times 2$ & Model number & 7LPL0678G8C1-1M01 \\
\hline & & Capacity & $5.3 \mathrm{Ah}$ \\
\hline & & Voltage & $25.2 \mathrm{~V}$ (7 cells) \\
\hline \multirow[t]{4}{*}{ Battery for control } & & Type & Lithium ion battery \\
\hline & $\times 2$ & Model number & $62 \mathrm{KSP} 545483-2$ \\
\hline & & Capacity & $5.5 \mathrm{Ah}$ \\
\hline & & Voltage & $22.2 \mathrm{~V}(6$ cells $)$ \\
\hline
\end{tabular}

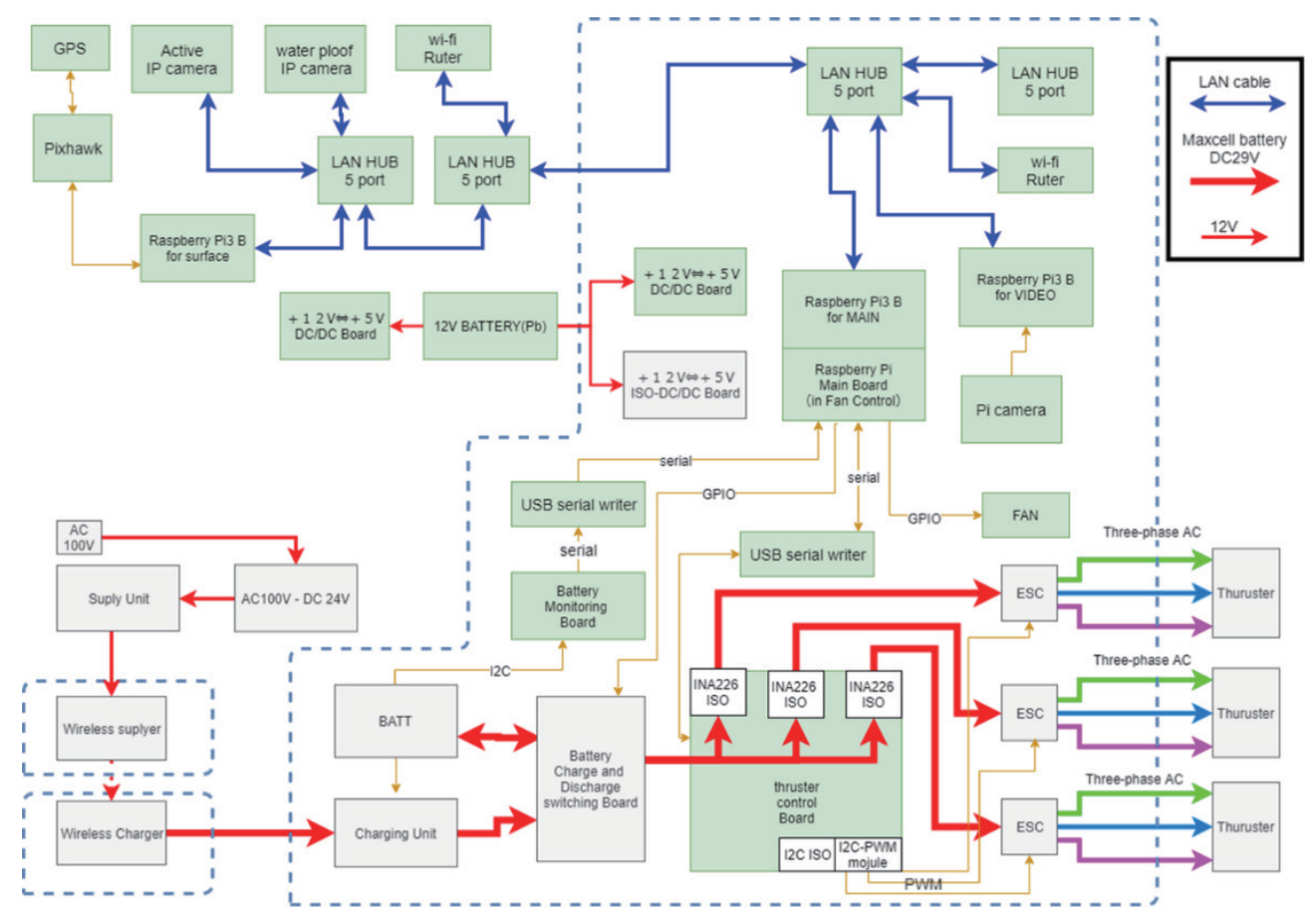

Fig. 2. (Color online) Configuration diagram of $\mu$-ASV control system. 


\section{3. $\mu$-ASV Model Identification}

Unlike in an ordinary ship, the hull angle of the $\mu$-ASV can be changed by a bow thruster alone as shown in Fig. 1. Namely, the travel distance of the $\mu$-ASV is controlled by the main thrusters after the hull angle is controlled by the bow thruster alone. In this case, ideally, the hull angle is not affected by the main thruster input and the travel distance is not affected by the bow thruster input. It is therefore considered that the travel distance and hull angle can be controlled independently by the main and bow thruster inputs, respectively. The motion models are identified on the basis of the following assumptions:

- The motion models are separated into the translational and rotational motion models.

- The main thruster input mainly contributes to the translational motion and the bow thruster input mainly contributes to the rotational motion.

When the translational motion system is denoted as $x_{1}$ and the rotational motion system is denoted as $x_{2}$, the motion model is described by Eqs. (1) and (2).

$$
\begin{gathered}
\boldsymbol{X}=\left[\begin{array}{l}
x_{1} \\
x_{2}
\end{array}\right], \boldsymbol{U}=\left[\begin{array}{l}
u_{1} \\
u_{2}
\end{array}\right], \boldsymbol{x}_{1}=\left[\begin{array}{c}
x \\
\dot{x}
\end{array}\right], \boldsymbol{x}_{2}=\left[\begin{array}{c}
\theta \\
\dot{\theta}
\end{array}\right] \\
\dot{\boldsymbol{X}}=\boldsymbol{A} \boldsymbol{X}+\boldsymbol{B} \boldsymbol{U}, \boldsymbol{A}=\left[\begin{array}{ll}
A_{11} & A_{12} \\
A_{21} & A_{22}
\end{array}\right], \boldsymbol{B}=\left[\begin{array}{ll}
B_{11} & B_{12} \\
B_{21} & B_{22}
\end{array}\right]
\end{gathered}
$$

Here, $x, \dot{x}, \theta$, and $\dot{\theta}$ are the absolute distance from the $\mu$-ASV, its translational velocity, the absolute angle of the $\mu$-ASV, and its rotational velocity, respectively. These equations are expanded into

$$
\begin{aligned}
& \dot{x}_{1}=A_{11} x_{1}+A_{12} x_{2}+B_{11} u_{1}+B_{12} u_{2}, \\
& \dot{x}_{2}=A_{21} x_{1}+A_{22} x_{2}+B_{21} u_{1}+B_{22} u_{2} .
\end{aligned}
$$

Here, it is considered that the main thruster input is dominant in the translational motion system, whereas the bow thruster input is dominant in the rotational motion system. When the terms involving $\left\{A_{12}, A_{21}\right\}$ and $\left\{B_{12}, B_{21}\right\}$, which are not directly affected by the input, are considered as disturbances, these terms can be expressed as $G_{1}(t)$ and $G_{2}(t)$. Then, Eqs. (5) and (6) are obtained.

$$
\begin{aligned}
& \dot{\boldsymbol{x}}_{1}=A_{11} x_{1}+B_{11} u_{1}+G_{1}(t), G_{1}(t)=A_{12} x_{2}+B_{12} u_{2} \\
& \dot{\boldsymbol{x}}_{2}=A_{22} x_{2}+B_{22} u_{2}+G_{2}(t), G_{2}(t)=A_{21} x_{1}+B_{21} u_{1}
\end{aligned}
$$

Because $G_{1}(t)$ and $G_{2}(t)$ are disturbances with a non-negligible effect on the motion of the $\mu$-ASV, sliding mode control, which is highly robust to disturbances, is used as a control 
technique. Thus, the models of the distance and rotation angle can be identified separately. As above, the control model's $x$ and $\theta$ can be obtained relatively easily by simply introducing a step input into the $\mu$-ASV and obtaining the response to the input. In the case of having different control inputs, it is also possible to design the sliding mode controllers separately. For example, although the response from the whole model is needed to use the proportionalintegral-derivative (PID) control method, it is known that the control input can be controlled separately using sliding mode control. ${ }^{(12)}$ Therefore, in this case, the control system can also be designed separately using sliding mode control as shown in Fig. $3 .{ }^{(9)}$ Both the translational and rotational motion systems of the $\mu$-ASV are considered to be second-order lag systems (RLC model). However, in this study, these motion systems are considered as first-order lag systems (RC model) to further simplify the control model. In the $\mu$-ASV, the transfer characteristics from the main thruster input to the translational velocity and those from the bow thruster input to the rotational velocity seem to correspond to those of second-order lag systems (RLC model). Namely, a hunting phenomenon may occur in the step response as shown in Fig. 4.
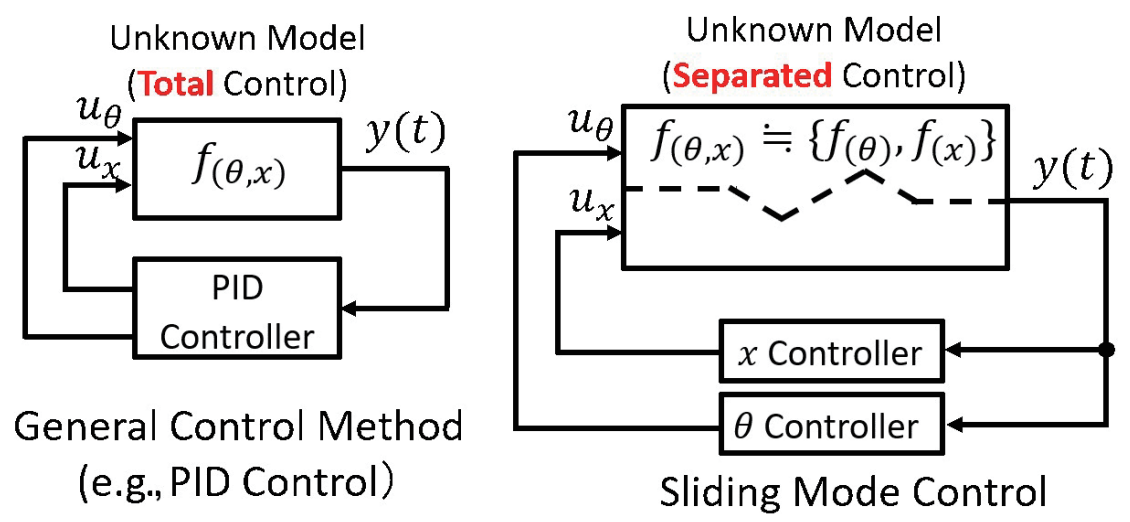

Fig. 3. (Color online) Configuration of control system using sliding mode control.

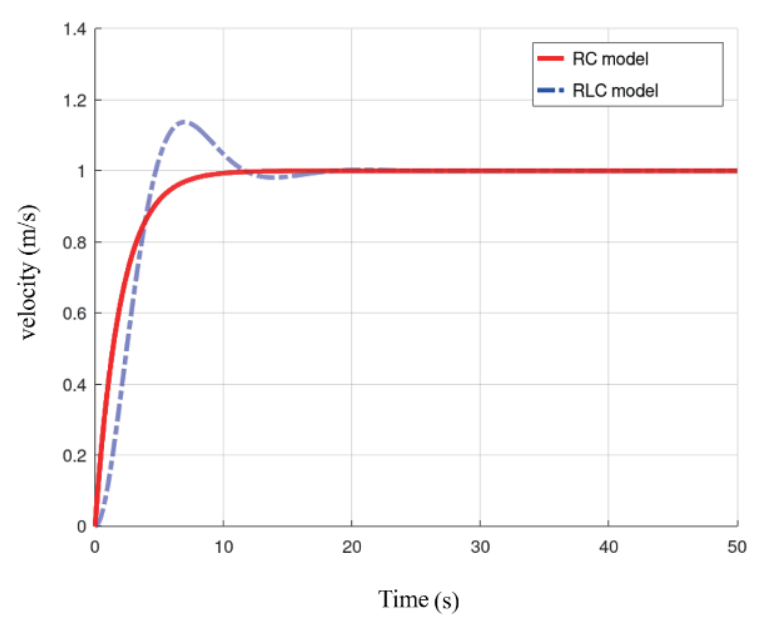

Fig. 4. (Color online) Example of simplified RLC model. 
Because it is difficult to identify such behavior accurately from the measured data including noise, we approximate the transfer characteristics by a first-order lag system (RC model) in this study. Although such a rough approximation is used for modeling, it is possible to obtain and outline the transfer characteristics. The control model describes the translational and rotational velocities of the $\mu$-ASV after sufficient time has passed. The actual motion of the $\mu$-ASV can be appropriately modeled when the $\mu$-ASV is moving slowly.

In this case, the model parameters of the translational motion system are the gain $E_{1}$ and time constant $\tau_{1}$, whereas the model parameters of the rotational motion system are the gain $E_{2}$ and time constant $\tau_{2}$. These parameters correspond to $A_{11}, A_{22}$, and $B_{11}$ in Eqs. (5) and (6) and are determined in this section.

\subsection{Identification of model of translational motion system}

The distance is measured using a radio frequency identification (RF-ID) tag (POZYX). Regarding the resolution performance, the largest positioning error is $\sim 0.1 \mathrm{~m}$. The velocity of the $\mu$-ASV is calculated using the distance data. The following is the calculation procedure.

(1) The position coordinates $(X, Y)$ of the $\mu$-ASV are evaluated from the data obtained using POZYX.

(2) The distance $L=\sqrt{x^{2}+y^{2}}$ is calculated.

(3) On the basis of the data of the distance $L, E$, and $\tau$ are identified using Eq. (8), which is obtained by integrating the first-order lag Eq. (7). Here, $\mathrm{E}$ is the gain factor, which is proportional to the pulse width modulation (PWM) input gain, and $\tau$ is the time constant of velocity.

(4) The first-order lag equation and actual measured values are evaluated.

The model of the translational motion system is identified by the above procedure. Figure 5 shows a graph of the position coordinates when the step response is given to the main thrusters.

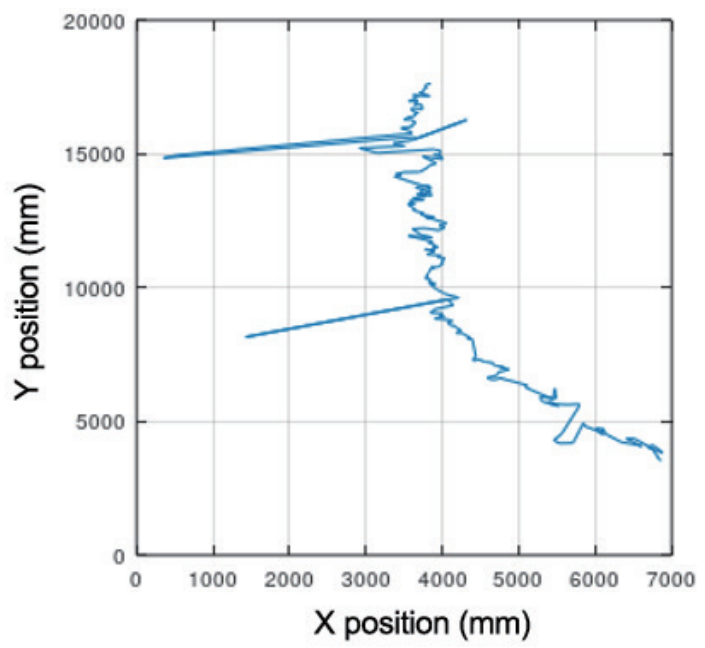

Fig. 5. (Color online) Position coordinates when step response is given to main thrusters. 
In general, the equation of motion for velocity is identified from the velocity data. However, obtaining the velocity data by differentiation and using the data to identify the model are difficult $^{(13)}$ because of large noise in the data as shown in this graph. Because the response is considered to be a first-order lag, as indicated in Eq. (5) and shown in Fig. 6, we use the technique of fitting the distance response to Fig. 6. Equation (7) is a general first-order lag equation and Eq. (8) is the integral of Eq. (7). $E$ and $\tau$ are identified by fitting Eq. (8) to Fig. 6.

$$
\begin{gathered}
\dot{L}(t)=E\left(1-e^{-\frac{1}{\tau} t}\right) \\
L(t)=E t+E \tau e^{-\frac{1}{\tau} t}+C
\end{gathered}
$$

Table 2 shows the results of the identification. Figure 7 shows the step response obtained using the identified parameters $E$ and $\tau$.

\subsection{Identification of model of rotational motion system}

The angle is measured using the Pixhawk accelerometer. The model is identified using the data of the rotation angular velocity. Figure 8 shows the graph of the rotation angular velocity

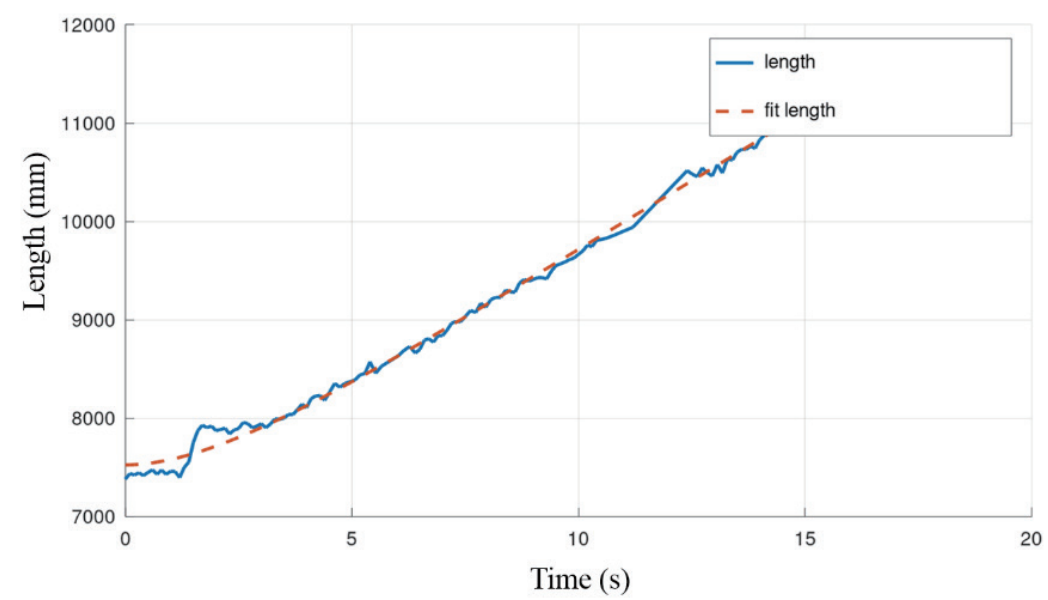

Fig. 6. (Color online) Example of distance response when step input is applied in distance direction of $\mu$-ASV (power: $400 \mathrm{~W})$.

Table 2

Approximate parameters obtained in translatory performance test.

\begin{tabular}{lcccc}
\hline Input gain & $42 / 256$ & $85 / 256$ & $128 / 256$ & Average \\
\hline Gain factor $E$ & 315.5 & 700.9 & 874.8 & 630.4 \\
$E /$ Input gain & 1923 & 2111 & 1750 & 1928 \\
Time constant $\tau(\mathrm{s})$ & 11.29 & 10.31 & 9.25 & 10.28 \\
\hline
\end{tabular}

Note: The input gain is the PWM (0-255) parameter given to the thrusters. 


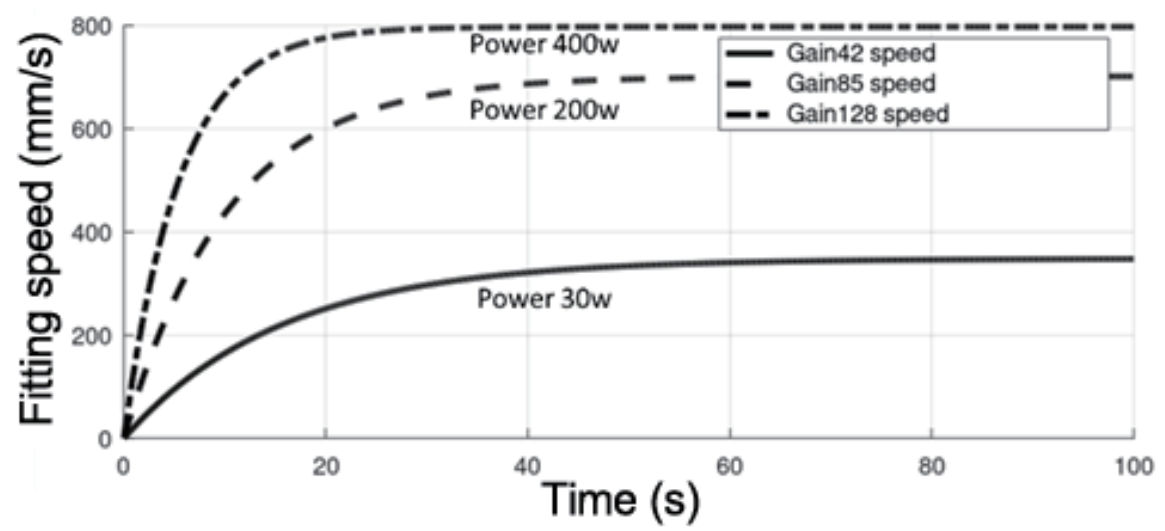

Fig. 7. Results of identification when step input is applied in distance direction of $\mu$-ASV.

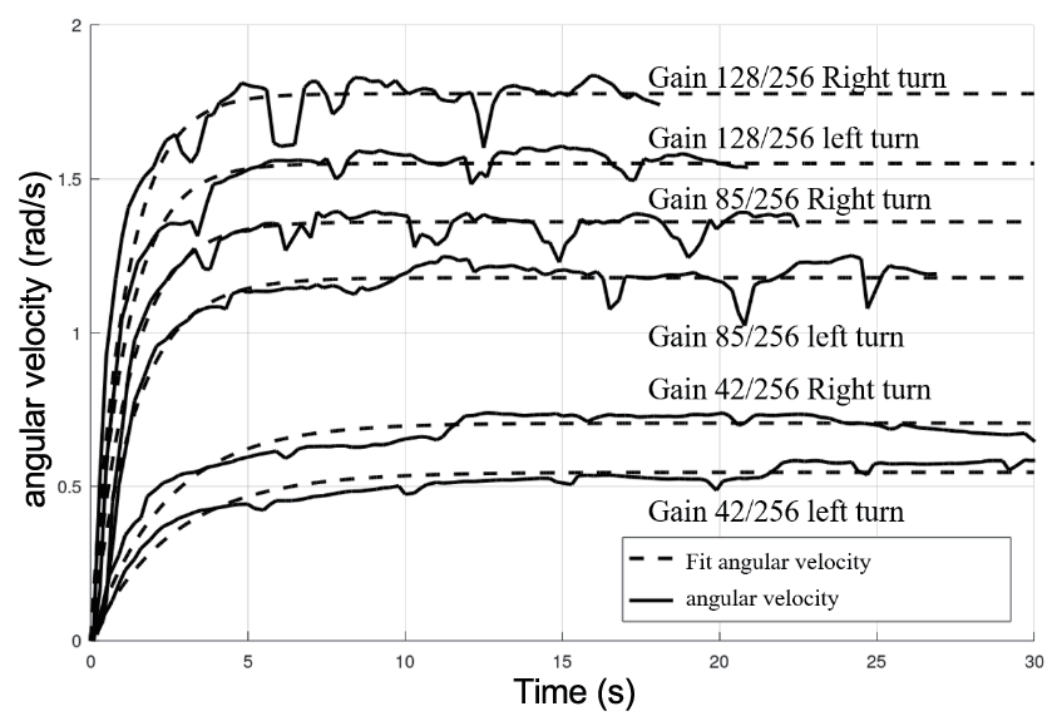

Fig. 8. Approximate data of angular velocity obtained in rotation performance test.

when the step response is given to the thruster. Parameters are identified assuming that the angular velocity is a first-order lag. The following is the calculation procedure.

(1) Obtain the rotation angle from Pixhawk.

(2) Obtain the differences between the angles and convert them into the angular velocity.

(3) Using the data of the angular velocity, identify $E$ and $\tau$ using the first-order lag Eq. (7).

(4) Evaluate the first-order lag equation and actual measured values.

Different from the identification of the model of the translational motion system, the model of the rotational motion system can be identified directly from the data of the angular velocity because of less noise in the data. Tables 3 and 4 show the identified parameters of the model. Figure 8 shows the step response obtained using the identified parameters $E$ and $\tau$. 
Table 3

Parameters obtained in rotation performance test (starboard).

\begin{tabular}{lcccc}
\hline Input gain & $42 / 256$ & $85 / 256$ & $128 / 256$ & Average \\
\hline Gain factor $E$ & 0.71 & 1.36 & 1.78 & 1.28 \\
$E /$ Input gain & 4.31 & 4.10 & 3.55 & 3.99 \\
Time constant $\tau(\mathrm{s})$ & 2.34 & 1.24 & 1.08 & 1.56
\end{tabular}

Table 4

Parameters obtained in rotation performance test (port).

\begin{tabular}{lcccc}
\hline Input gain & $42 / 256$ & $85 / 256$ & $128 / 256$ & Average \\
\hline Gain factor $E$ & 0.55 & 1.18 & 1.55 & 1.09 \\
E/Input gain & 3.33 & 3.55 & 3.10 & 3.33 \\
Time constant $\tau(\mathrm{s})$ & 2.58 & 1.42 & 1.11 & 1.70
\end{tabular}

\section{Simulation on ROS}

In the simulation, Gazebo was used as a physical simulator and ROS was used to create the simulation model. ${ }^{(9,10)}$ They are both open-source software programs that operate on Linux. ROS is the world's most commonly used robotic software and provides a library and tools for assisting the development of robots and controllers. Gazebo is a three-dimensional robot simulator that can use multiple physical engines and is fully compatible with ROS. The simulation was conducted using Gazebo and the parameters identified above. Because Gazebo cannot simulate the physical movement of water when there is power transmission through water, we conducted the DPS simulation by assuming that the $\mu$-ASV is a mobile truck. Omni wheels were used to simulate the ship model (Fig. 9).

The physical model parameters of the truck were determined by referring to the models identified in Sect. 3. The sliding mode control system was developed as follows:

- The equivalent linear sliding mode control was used to control the distance.

- The basic sliding mode control was used to control the rotation.

The reasons for using different sliding mode control methods are as follows. We would like to control the distance of the $\mu$-ASV precisely using the equivalent linear sliding mode control to avoid chattering (going back and forth). On the other hand, we would like to maintain the prescribed angle in the heading control even if chattering may occur.

For the switching gain $k$, hyperplane $S$, and saturation parameter $\alpha$, which are control parameters, two suboptimal solutions (GA1 and GA2) were obtained with $\mathrm{GA}^{(10)}$ using the ROS and GAZEBO Simulation.

\subsection{Identification of model of translational motion system}

The position control using the main thrusters is Eq. (9), in which the state space model of an $\mathrm{RC}$ circuit is extended to a third-order servo system. $R d$ is a servo value. Chattering can be prevented by using the equivalent linear input. We would like to avoid chattering back and forth in the control of the $\mu$-ASV.

In addition to the control model of the first-order response identified above, we examined the second-order lag element, which seems to have a negligible effect, to create the equivalent linear input. Here, the state variable is

$$
\boldsymbol{X}_{1}=\left[\begin{array}{c}
x \\
\dot{x} \\
\int x d t
\end{array}\right], \boldsymbol{X}_{L}=\left[\begin{array}{c}
x \\
\dot{x}
\end{array}\right]
$$




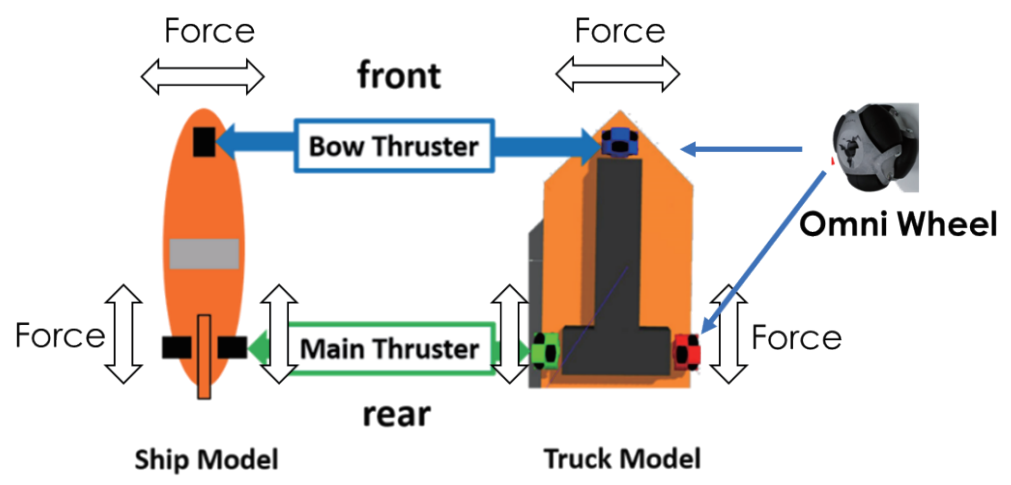

Fig. 9. (Color online) Schematic of truck model.

The equation of the $\mu$-ASV's motion is

$$
\dot{\boldsymbol{X}}_{L}=\left[\begin{array}{cc}
0 & 1 \\
A_{L_{21}} & -\frac{1}{\tau}
\end{array}\right]\left[\begin{array}{l}
x \\
\dot{x}
\end{array}\right]+\left[\begin{array}{c}
0 \\
\frac{1}{E}
\end{array}\right] u, \boldsymbol{A}_{L}=\left[\begin{array}{cc}
0 & 1 \\
A_{L_{21}} & -\frac{1}{\tau}
\end{array}\right], \boldsymbol{B}_{L}=\left[\begin{array}{c}
0 \\
\frac{1}{E}
\end{array}\right] .
$$

Therefore, the equation including the servo value of the servo model is

$$
\left\{\begin{array}{l}
\dot{\boldsymbol{X}}_{1}=\boldsymbol{A}_{11} \boldsymbol{X}_{1}+\boldsymbol{B}_{11} u_{1}+\boldsymbol{D} \cdot R d \\
\boldsymbol{A}_{11}=\left[\begin{array}{cc}
0 & A_{L} \\
-1 & 0
\end{array}\right], \boldsymbol{B}_{11}=\left[\begin{array}{c}
B_{L} \\
0
\end{array}\right], \boldsymbol{D}=\left[\begin{array}{l}
0 \\
1
\end{array}\right] .
\end{array}\right.
$$

$\boldsymbol{A}_{L_{21}}$, which is the parameter of the acceleration system, is not determined in Sect. 3. However, $\boldsymbol{A}_{L_{21}}$ is sufficiently smaller than the identified values of $\tau$ and $1 / E$ and has a smaller effect on the motion and control. In the design of the equivalent linear input, therefore, these values were considered to not change in polarity, which determines the stability. In practice, the hyperplane $S$, switching gain $K$, and saturation function $\alpha$ are designed using GA. The switching hyperplane is represented as

$$
\boldsymbol{S}^{1}=\left[\begin{array}{lll}
S_{12} & S_{12} & -1
\end{array}\right]
$$

The equivalent linear input including the servo system is

$$
\begin{gathered}
u_{e q}=-\left(\boldsymbol{S}^{1} \boldsymbol{B}_{11}\right)^{-1} \boldsymbol{S}^{1} \boldsymbol{A}_{11} \boldsymbol{X}_{1}-\left(\boldsymbol{S}^{1} \boldsymbol{B}_{11}\right)^{-1} \boldsymbol{S}^{1} D \cdot R d, \\
u_{e q} \fallingdotseq-\frac{E}{S_{12}}\left[\begin{array}{lll}
1 & S_{12} A_{L_{22}} & \left.\frac{S_{11}-S_{12}}{\tau}\right] \boldsymbol{X}_{1}-\frac{E}{S_{12}} S_{13} \cdot R d .
\end{array}\right.
\end{gathered}
$$


When $\boldsymbol{A}_{L_{12}}=0$ is not considered because it is sufficiently small, the following equation is obtained:

$$
u_{e q} \fallingdotseq-\frac{1}{S_{11} \boldsymbol{B}_{L}}\left[\begin{array}{lll}
1 & S_{11} A_{12} & S_{2} A_{23}
\end{array}\right] \boldsymbol{X}_{1}-\frac{1}{S_{11} \boldsymbol{B}_{L}} S_{13} \cdot R d .
$$

Here, the controller is represented as

$$
\left\{\begin{array}{l}
u_{1}=u_{e q}-K_{1} \cdot \operatorname{sat}\left(\alpha_{1}, \sigma_{1}\right) \\
\operatorname{sat}\left(\alpha_{1}, \sigma_{2}\right)=\frac{\sigma_{1}}{\left|\sigma_{1}\right|+\alpha_{1}} \\
\sigma_{1}=S_{1} \cdot X_{1} \\
\boldsymbol{X}_{1}=\left[\begin{array}{lll}
x & \dot{x} & \int x d t
\end{array}\right], S^{1}=\left[\begin{array}{lll}
S_{11} & S_{12} & -1
\end{array}\right] .
\end{array}\right.
$$

\subsection{Design of angle controller}

The angle control by the bow thruster was not extended to the servo system. The proportional control was used to follow the target value. This is because the heading control of a ship is affected by a number of disturbances. We therefore used the simple sliding mode control, which is easily designed and highly robust despite the large amount of chattering.

The given state quantity $\boldsymbol{X}_{2}$ consists of the difference $\left(\theta-\theta_{r}\right)$ between the target angle $\left(\theta_{r}\right)$ and the present angle $(\theta)$ as well as the angular velocity $(\dot{\theta})$ in the direction of the model yaw angle (around the $z$-axis of the model). Here, the control system is represented as

$$
\left\{\begin{array}{l}
u_{2}=-K_{2} \cdot \operatorname{sat}\left(\alpha_{2}, \sigma_{2}\right) \\
\operatorname{sat}\left(\alpha_{2}, \sigma_{2}\right)=\frac{\sigma_{2}}{\left|\sigma_{2}\right|+\alpha_{2}} \\
\sigma_{1}=S^{2} \cdot X_{1} \\
\boldsymbol{X}_{2}=\left[\begin{array}{ll}
\theta-\theta_{r} & \dot{\theta}
\end{array}\right]^{t}, S^{2}=\left[\begin{array}{ll}
S_{21} & 1
\end{array}\right] .
\end{array}\right.
$$

\subsection{Simulation results}

The parameters of the optimal controller were calculated on ROS using the GA. Tables 5 and 6 show the obtained parameters. Figures 10 and 11 show the results of the simulation using these parameters. Distance control and angle control were successfully achieved using the simple DPS. However, we could not find the control parameters that accomplish the two conflicting goals, namely, distance control and angle control. In practice, it is desirable to use GA2 parameters when emphasizing heading control and GA1 parameters when emphasizing position control. Although heading control was achieved by the simple sliding mode control alone in this simulation, it is desirable to apply the equivalent linear sliding mode control to heading control as in the case of position control. 
Table 5

Parameters for distance control with simple DPS.

\begin{tabular}{lccc}
\hline & & GA1 & GA2 \\
\hline & $K_{1}$ & $1.13 \times 10^{-3}$ & $1.26 \times 10^{-3}$ \\
Distance & $\alpha_{1}$ & $5.49 \times 10^{-1}$ & $1.62 \times 10^{-5}$ \\
control & $S_{11}$ & $6.81 \times 10^{-2}$ & $4.25 \times 10^{-3}$ \\
& $S_{12}$ & $5.74 \times 10^{-4}$ & $5.98 \times 10^{-2}$ \\
\hline
\end{tabular}

Table 6

Parameters for angle control with simple DPS.

\begin{tabular}{lccc}
\hline & & GA1 & GA2 \\
\hline & $K_{2}$ & 20.59 & 11.84 \\
Angle & $\alpha_{2}$ & $2.15 \times 10^{-2}$ & $3.14 \times 10^{-1}$ \\
control & $S_{21}$ & 26.24 & 24.94 \\
& $\alpha_{1}$ & $5.49 \times 10^{-1}$ & $1.62 \times 10^{-5}$ \\
\hline
\end{tabular}

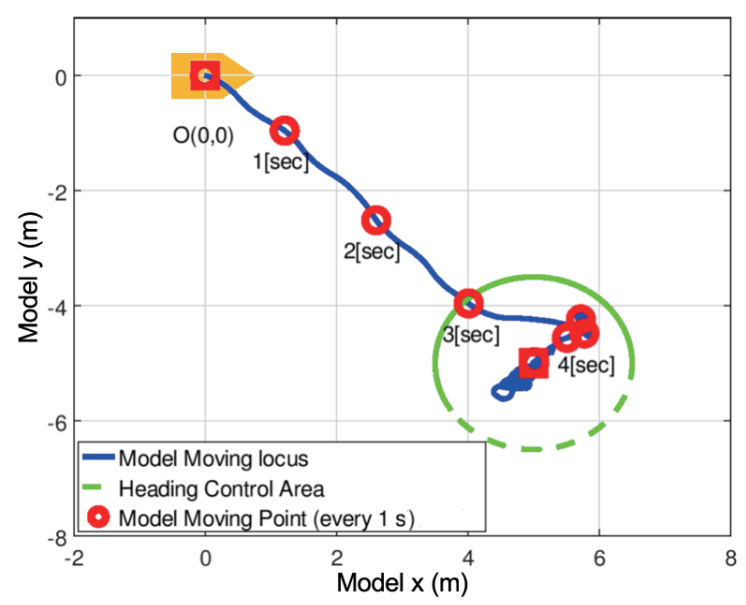

Fig. 10. (Color online) Locus of model when GA1 parameters are used.

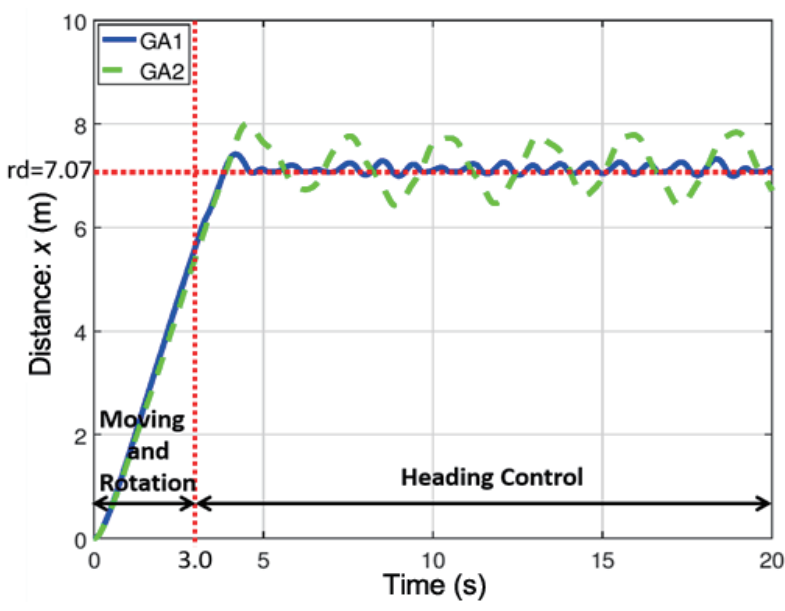

(a)

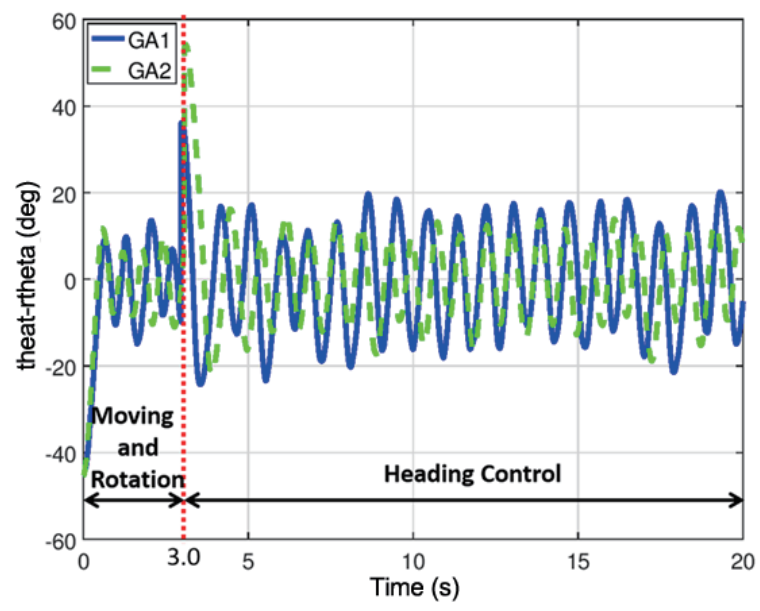

(b)

Fig. 11. (Color online) Time response of distance and angle when controlled by simple DPS.

\section{Open Sea Test}

The $\mu$-ASV was tested in the open sea (Matsukawa-ura, Minamisouma City, Fukushima Prefecture). Because the automatic control and DPS control were not implemented, the $\mu$-ASV was tested in the manual mode using a joystick. ${ }^{(14,15)}$ The $\mu$-ASV showed good motion performance in the open sea without any problems. The Wi-Fi communication, cameras, and sensors such as the IMU worked properly. Figure 12 shows the track chart of the $\mu$-ASV in 


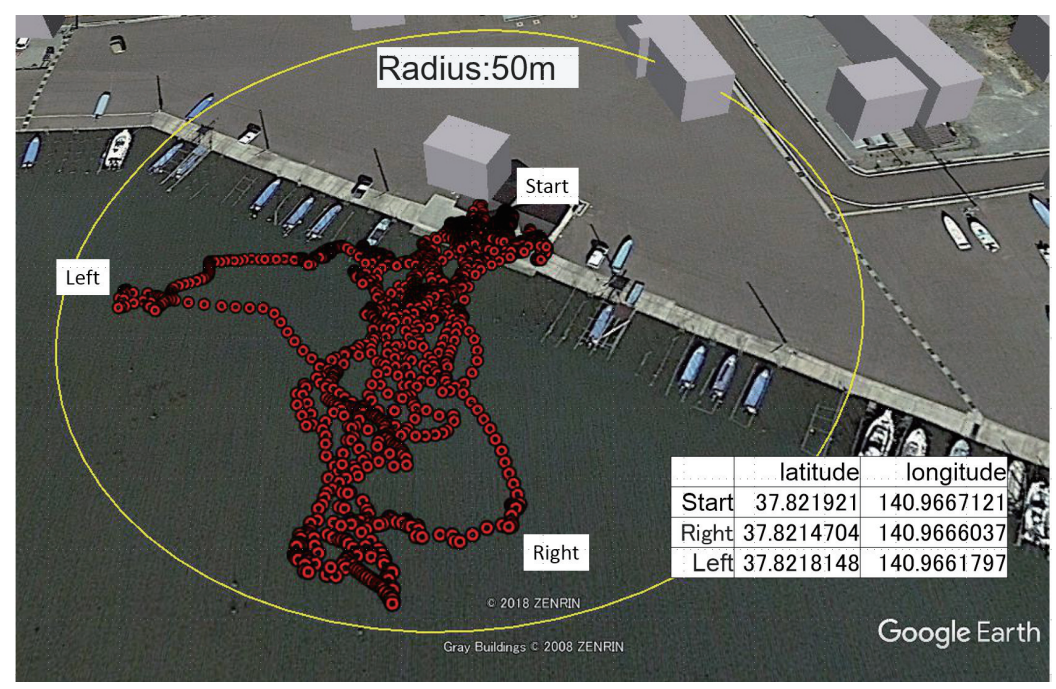

Fig. 12. (Color online) Results of manual operation test.

this test. The $\mu$-ASV implements the sliding control method. The simulation results must be implemented in the sliding mode control system in the future.

\section{Conclusions}

We conducted the modeling of a $\mu$-ASV. The ROS model of the $\mu$-ASV was created by applying the parameters identified in this study to a mobile truck that had omni wheels instead of thrusters. By using ROS, distance control and angle control were successfully achieved using sliding mode control. Also, control with a simple DPS was achieved. The $\mu$-ASV was tested in the open sea. Our next step is to perform sensing using GPS and Pixhawk (IMU) and achieve the control on site with the simple DPS using the sliding mode control. It is also expected that the $\mu$-ASV will be used for automated investigations in radiation-contaminated areas of Fukushima Prefecture as it is in cooperation with "MAINAMI". Now, the ASV of MAINAMI is being developed by the Japan Atomic Energy Agency (JAEA), Japan Agency for Marine-Earth Science and Technology (JAMSTEC), and Tokyo University of Marine Science and Technology (TUMSAT). The $\mu$-ASV and MAINAMI were demonstrated at the Advanced Fisheries Technology Experience Fair (2019/08/04).

\section{Acknowledgments}

This study was supported by a Grant-in-Aid for Research from the Fundamental Research Developing Association for Shipbuilding and Offshore (REDAS) and the President's Discretionary Expenses of Tokyo University of Marine Science and Technology. The batteries were provided by Maxell. The open sea test was conducted with the cooperation of JAMSTEC, JAEA, and Bandai Marine. We sincerely appreciate their support. 


\section{References}

1 J. Xu, M. Wang, and L. Qiao: Ocean Eng. 105 (2015) 54.

2 A. Martorell-Torres, M. Massot-Campos, E. Guerrero-Font, and G. Oliver-Codina: Proc. IFAC-PapersOnLine 51[29] (2018) 147.

3 T. I. Fossen: Handbook of Marine Craft Hydrodynamics and Motion Control (John Wiley \& Sons, Ltd., Wiley, 2011).

4 N. Mizuno and S. Matsumoto: Proc. 9th IFAC Conf. Control Applications in Marine Systems (2013) 17.

5 M. Bibuli, G. Bruzzone, M. Caccia, G. Ippoliti, S. Longhi, G. Orlando, and G. M. Pelusi: Proc. 9th IFAC Conf. Manoeuvring and Control of Marine Craft (2012) 19.

6 M. Bibuli, G. Bruzzone, M. Caccia, A. Caiti, and N. Di Lecce: Proc. 8th IFAC Conf. Control Applications in Marine Systems (2010) 15.

7 G. Conte, G. P. De Capua, and D. Scaradozzi: Proc. 9th IFAC Conf. Manoeuvring and Control of Marine Craft (2012) 19.

8 A. J. Healey and D. Lienard: IEEE J. Oceanic Eng. 18 (1993) 327.

9 K. Nakamura, J. Tahara, M. Koike, F. Zhang, and M. Saito: 23rd Proc. Int. Symp. Artificial Life and Robotics (2018) 143.

10 K. Nakamura, J. Tahara, M. Koike, F. Zhang, and M. Saito: 24th Proc. Int. Symp. Artificial Life and Robotics (2019) 172 .

11 I. S. Silva, F. Campopiano, G. S. V. Lopes, A. K. Uenojo, H. T. Silva, E. L. Pellini, A. A. Alvarez, and E. A. Barros: Proc. IFAC-PapersOnLine 51[29] (2018) 8.

12 V. Utkin: Sliding Mode Control in Electro-Mechnical Systems (Taylor \& Francis, Boca Raton, 2009).

13 O. Köckritz, H. Korte, and K.-J. Windeck: Proc. IFAC-PapersOnLine 51[29] (2018) 195.

14 G. Peeters, R. Boonen, M. Vanierschot, M. DeFilippo, P. Robinette, and P. Slaets: Proc. IFAC-PapersOnLine 51[29] (2018) 207.

15 J. Park, J. Jung, Y. Lee, J. Chae, Y. Lee, and J. Choi: Proc. IFAC-PapersOnLine 51[29] (2018) 165.

\section{About the Authors}

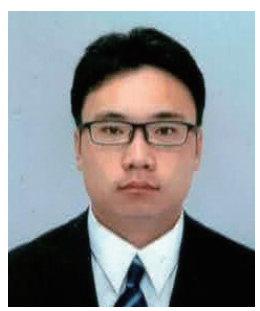

Yamato Kawamura is currently an M. Eng. student of Tokyo University of Marine Science and Technology. In particular, he is interested in the automatic control of ASVs and the operation of ROVs.

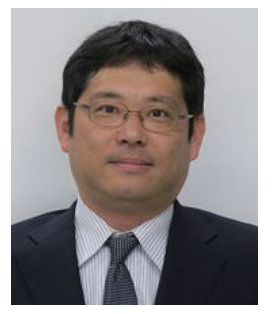

Junichiro Tahara received his B. Eng. and M. Eng. degrees from Tokyo University of Mercantile Marine in 1993 and 1995, respectively, and his Dr. Eng. degree from Tokyo University of Mercantile Marine in 2002. From 1995 to 2003, he was an associate professor at Tokyo Metropolitan College of Technology, Japan. From 2003 to 2015, he was a researcher at JAMSTEC. Since 2015, he has been an associate professor at Tokyo University of Marine Science and Technology. His research interests are in nonlinear vibration control and marine robots. 


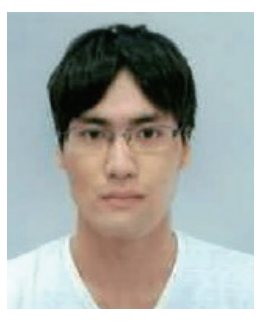

Tetsu Kato is currently an M. Eng. student of Tokyo University of Marine Science and Technology. He is now studying at Shanghai Ocean University. $\mathrm{He}$ is interested in the automatic control of ASVs and unmanned vehicles.

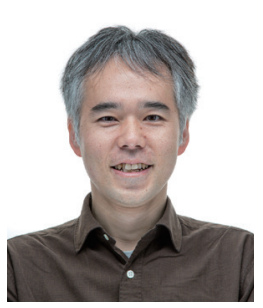

Shoichiro Baba received his B. Eng. and M. Eng. degrees from Osaka City University in 1995 and 1997, respectively. From 1997 to 2008, he was a researcher at IHI Corporation. Since 2008, he has been an engineer at the Japan Agency for Marine-Earth Science and Technology. He is engaged in the development of an oceanographic observation system that can monitor global environmental changes. Also, he is interested in a calibration system for oceanographic thermometers that can be traceable to national standards.

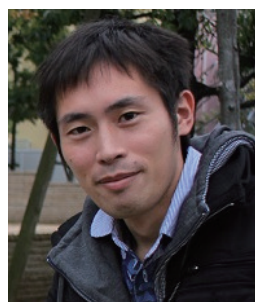

Masakazu Koike received his M.S. degree in engineering from Tokyo Institute of Technology, Japan, in 2006, and his Ph.D. degree in engineering from Shinshu University, Japan, in 2013. From 2013 to March 2015, he was a Ph.D. researcher at the Department of Mechanical and Environmental Informatics, Graduate School of Information Science and Engineering, Tokyo Institute of Technology. Since April 2015, he has been an assistant professor at the Department of Marine Electronics and Mechanical Engineering, Tokyo University of Marine Science and Technology. His research interests include the development of control theory for pneumatic isolation tables. 